\title{
Speed and Rotor Resistance Estimation of AC Induction Motor
}

\author{
Wang Dafang ${ }^{1}$, Wang Miaoran ${ }^{1}$, Dong Guanglin ${ }^{1}$, Wei Hui ${ }^{1}$ and Xu Zexu ${ }^{1}$ \\ ${ }^{I}$ School of Automotive Engineering, Harbin Institute of Technology, 264209 Weihai, China
}

\begin{abstract}
The speed estimation method based on Extended Kalman Filter (EKF) is widely used in speed sensorless induction motor. The method has high accuracy and good robustness. However, EKF is sensitive to parameters. In particular, the rotor resistance becomes up to twice the original when the motor is running. This greatly affects the speed identification accuracy. This paper achieves online estimation of rotor resistance utilizing the Model Reference Adaptive Controller (MRAC) based on reactive power. This ensures that the speed is estimated with high accuracy even if the rotor resistance changes suddenly. Simulation results have been presented to verify the effectiveness of the method.
\end{abstract}

\section{$1 \quad$ INTRODUCTION}

Speed estimation methods of AC induction motor mainly include motor anisotropy-based method and motor model-based method.

The motor anisotropy-based method is not sensitive to motor parameters, but requires high measurement accuracy and additional hardware.

The motor model-based method mainly includes: direct calculation method [1], model reference adaptive system-based method, observer-based speed estimation method and intelligent control method. This class of method is sensitive to motor parameters because it is based on motor model. The direct calculation method is sensitive to load and poor in anti-interference. When the reference model of Model Reference Adaptive System (MRAS) contains other motor parameters, it will greatly affect the recognition accuracy. The intelligent control method requires additional hardware. It is computationally intensive and still at the stage of theoretical research. Observer-based speed estimation method is mainly comprised of full-order adaptive state observer, EKF and sliding mode observer method. The full-order adaptive state observer is not stable in the lowspeed power generation area. The sliding mode observer has a problem of jitter, which restricts its use. EKF is more accurate and has been widely used in speed sensorless induction motors.

There exists many methods for online estimation of rotor resistance, such as signal injection-based method, MRAS [2]-[3], least squares method, model observation method and intelligent control method. The least squares method is sensitive to measuring noise and changes in speed. And it is poor in anti-interference. Model observation method is complex in calculation and it is difficult to select the gain of the observer. If the observation gain is not properly selected, it will cause system instability. The reference model of reactive power-based MRAS is independent of stator resistance and is very popular. This method is simple, small in computation, easy to implement and has good real-time performance.

This paper utilizes EKF to estimate motor speed. This method is sensitive to motor parameters, especially rotor resistance. Therefore, it is necessary to add online identification of the rotor resistance. This paper utilizes reactive power-based MRAS method to achieve online estimation in order to ensure the speed estimation accuracy.

\section{SPEED ESTIMATION OF AC INDUCTION MOTOR}

This paper uses EKF to estimate the speed of $\mathrm{AC}$ induction motor. The EKF linearizes complex nonlinear systems such as induction motors by removing highorder terms. This linearization is only a sub-optimal approximation of the original nonlinear system and it may cause errors. But these errors can be included in the system noise or measurement noise to overcome the linearization deficiencies. After the induction motor is linearized, the rotor speed is derived by derivation.

\subsection{Basic Principles Of Extended Kalman Filter Theory}

Assume that the nonlinear discrete system is as follows:

$$
\left\{\begin{array}{l}
x(k+1)=f(x(k), u(k)) \\
y(k+1)=h(x(k+1))
\end{array}\right.
$$


where $x(k)$ is the state variable. $u(k)$ is the input variable. $y(k+1)$ is the output variable.

The EKF algorithm is relatively mature and its iteration process is as follows:

Set initial values, such as: system noise, measurement noise.

State prediction: Use the kth state estimate value $\hat{x}(k)$ to calculate the $\mathrm{k}+1$ state prediction value $\tilde{x}(k+1)$. Update the Jacobian matrix:

$$
G(k+1)=\left.\frac{\partial f(x(k+1), u(k+1))}{\partial x}\right|_{x(k+1)=\tilde{x}(k+1)}
$$

Estimate the error covariance matrix:

$$
\tilde{P}(k+1)=G(k+1) \hat{P}(k) G^{T}(k+1)+Q_{k}
$$

where $Q_{k}$ is the system noise matrix.

Update the Hessians of the output matrix:

$$
H(k+1)=\left.\frac{\partial h(x(k+1))}{\partial x}\right|_{x(k+1)=\tilde{x}(k+1 \mid k)}
$$

Calculate the Kalman gain matrix:

$$
K(k+1)=\tilde{P}(k+1) H^{T}\left[H \tilde{P}(k+1) H^{T}+R_{k}\right]^{-1}
$$

State Estimation:

$$
\hat{x}(k+1)=\tilde{x}(k+1)+K[y(k+1)-h(\tilde{x}(k+1))]
$$

where $y(k+1)$ is the measurement vector.

Calculate the error covariance matrix:

$$
\hat{P}(k+1)=\tilde{P}(k+1)-K(k+1) H(k+1) \tilde{P}(k+1)
$$

\subsection{Rotation Speed Estimation Based on EKF}

The stator current, rotor flux, and motor speed are selected as state variables to establish a continuous state equation of the $\mathrm{AC}$ induction motor in the two-phase stationary coordinate system. In order to complete programming in the embedded system, we discretized the continuous state equation. After removing the high-order items in the system matrix and the input matrix, the discrete state equations of $\mathrm{AC}$ induction motor in the two-phase stationary coordinate system are as follows:

$$
\left\{\begin{array}{l}
x(k+1)=A^{d}(k) x(k)+B^{d} u(k) \\
y(k+1)=C^{d} x(k+1)
\end{array}\right.
$$

where $A^{d}(k)$ is the discrete system matrix. $B^{d}$ is the discrete input matrix. $C^{d}$ is the discrete output matrix.

$$
\begin{aligned}
& A^{d}(k)=\left[\begin{array}{ccccc}
1-T_{s} \eta & 0 & T_{s} \lambda / \tau_{r} & T_{s} \lambda w_{r}(k-1) & 0 \\
0 & 1-T_{s} \eta & -T_{s} \lambda w_{r}(k-1) & T_{s} \lambda / \tau_{r} & 0 \\
T_{s} L_{m} / \tau_{r} & 0 & 1-T_{s} / \tau_{r} & -T_{s} w_{r}(k-1) & 0 \\
0 & T_{s} L_{m} / \tau_{r} & T_{s} w_{r}(k-1) & 1-T_{s} / \tau_{r} & 0 \\
0 & 0 & 0 & 0 & 1
\end{array}\right] \\
& B^{d}=\left[\begin{array}{cc}
\frac{T_{s}}{\sigma L_{s}} & 0 \\
0 & \frac{T_{s}}{\sigma L_{s}} \\
0 & 0 \\
0 & 0 \\
0 & 0
\end{array}\right], C^{d}=\left[\begin{array}{ll}
1 & 0 \\
0 & 1 \\
0 & 0 \\
0 & 0 \\
0 & 0
\end{array}\right]^{T}, \bar{x}=\left[\begin{array}{l}
i_{\alpha}(t) \\
i_{\beta}(t) \\
\psi_{\alpha}(t) \\
\psi_{\beta}(t) \\
\omega_{r}(t)
\end{array}\right] \\
& u=\left[\begin{array}{ll}
u_{\alpha}(t) & u_{\beta}(t)
\end{array}\right]^{T}, y=\left[\begin{array}{ll}
i_{\alpha}(t) & i_{\beta}(t)
\end{array}\right]^{T} \tau_{r}=L_{r} / R_{r} \\
& , \eta=\left(R_{s} L_{r}^{2}+R_{r} L_{m}^{2}\right) /\left(L_{r}^{2} L_{s} \sigma\right), \lambda=L_{m} /\left(\sigma L_{s} L_{r}\right)
\end{aligned}
$$

Only by adding the system matrix update $A^{d}(k+1)$ before the state prediction, the EKF algorithm can be used to estimate the motor speed.

\section{ROTOR RESISTANCE ONLINE ESTIMATION OF AC INDUCTION MOTOR}

Due to the skin effect and saturation level caused by temperature and frequency, motor parameters will change In particular, the rotor resistance becomes up to twice the original when the motor is running. EKF is sensitive to motor parameters. Rotor resistance changes will produce speed estimation error, and even make motor run unstable. Therefore, to ensure accuracy of the speed estimation, it is necessary to perform online estimation of the rotor resistance.

\subsection{Basic Principles of MRAS}

MRAS is mature and has been widely used in motor control. It consists of a reference model, an adjustable model and an adaptive mechanism. The outputs of the two models are the same physical variables. The difference of the outputs is subjected to the adaptive mechanism to obtain the estimated variable. The estimated physical variable is brought back to the adjustable model to achieve real-time tracking to the reference model. The MRAS block diagram is as follows:

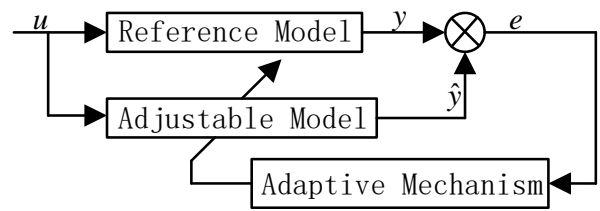

Figure 1: Model reference adaptive block diagram 


\subsection{Rotor Resistance Online Estimation Based On MRAS}

MRAS based on flux observation is more commonly used. Its reference model is the voltage model with stator resistance. When the motor is running, the stator resistance will change with temperature. This results in inaccuracy of the flux observed by reference model. Therefore, the rotor resistance is inaccurate. The reference model of reactive power-based MRAS does not consist of stator resistance. It can avoid the effect of stator resistance, thereby improving the accuracy of rotor resistance.

The reference model is instantaneous reactive power:

$$
Q_{r e f}=v_{q s} i_{d s}-v_{d s} i_{q s}
$$

where $v_{d s}\left(v_{q s}\right)$ is the $\mathrm{d}(\mathrm{q})$ component of the stator voltage vector. $i_{d s}\left(i_{q s}\right)$ is the $\mathrm{d}(\mathrm{q})$ component of the stator current vector.

The adjustable model is steady-state reactive power:

$$
Q_{a d j}=\sigma L_{s} w_{e}\left(i_{d s}^{2}+i_{q s}^{2}\right)+w_{e} \frac{L_{m}^{2}}{L_{r}} i_{d s}^{2}
$$

where $L_{s}$ is the stator inductance. $L_{m}$ is the mutual inductance. $L_{r}$ is the rotor inductance. $w_{e}$ is the synchronously rotating angular velocity. $\sigma=1-L_{m}^{2} /\left(L_{s} L_{r}\right)$ is the total leakage factor.

The error is obtained by subtracting the steady state reactive power from the instantaneous reactive power. It is used as the input to the proportional-integral control. And the output is the change in the rotor resistance, which can then be applied to the real-time adjustment of rotor resistance.

\section{SIMULATION}

\subsection{Speed and Rotor Resistance Estimation Block Diagram}

The block diagram of EKF-based speed estimation and MRAS-based rotor resistance estimation for indirect field oriented controlled (IFOC) induction motor (IM) is as follows:

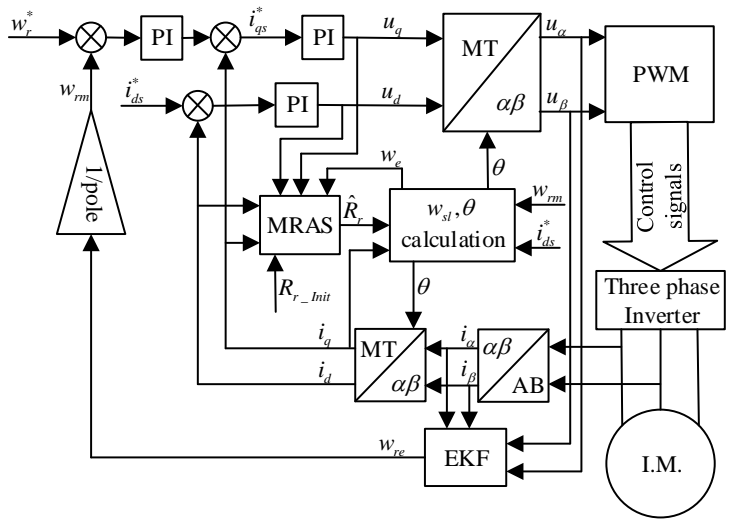

Figure 2: The block diagram of EKF-based speed estimation and MRAS-based rotor resistance estimation for IFOC IM

\subsection{Simulation Results and Analysis}

In order to verify the effectiveness of the speed and rotor resistance estimation method, a simulation model is constructed according to the block diagram of FIG. 2.

Simulation conditions are as follows: Setting the mechanical speed to $1000 \mathrm{r} / \mathrm{min}, 300 \mathrm{r} / \mathrm{min}, 60 \mathrm{r} / \mathrm{min} ; \mathrm{t}=$ $0.1 \mathrm{~s}$, giving the load of $10 \mathrm{Nm} ; \mathrm{t}=0.6 \mathrm{~s}$, the rotor time constant changing from 1.083 to $1.5 ; \mathrm{t}=1.2 \mathrm{~s}$, adding the rotor resistance online estimation; setting simulation time to $2 \mathrm{~s}$. The identification results are shown from FIG. 3 to FIG. 5 .
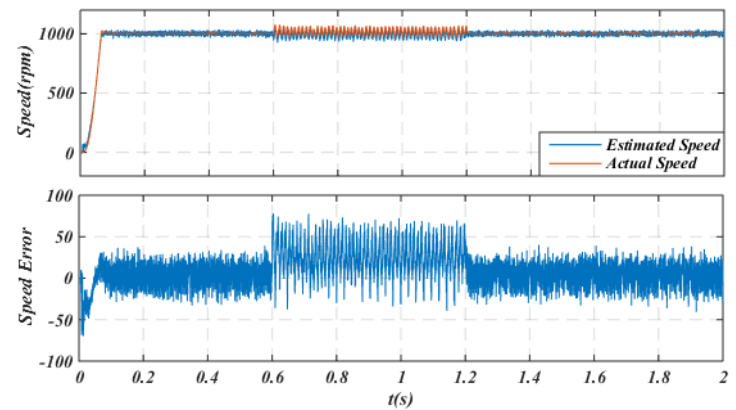

Figure 3: $1000 \mathrm{r} / \mathrm{min}$ speed and speed error waveform

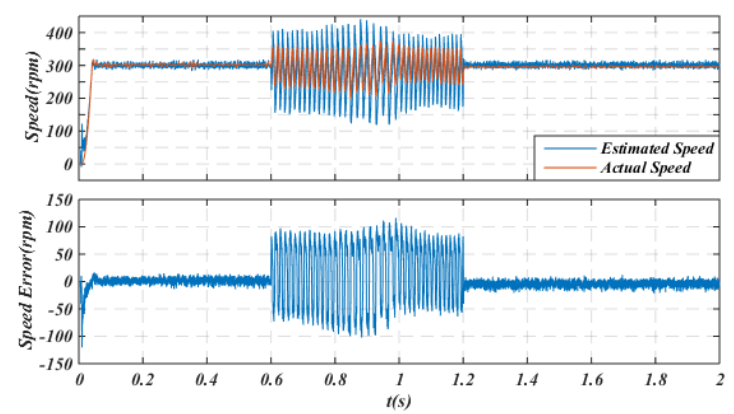

Figure 4: 300r/min speed and speed error waveform 


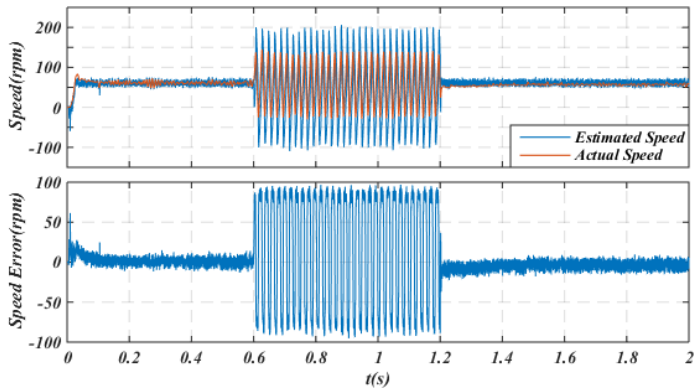

Figure 5: 60r/min speed and speed error waveform

From Fig. 3 to Fig. 5, it can be seen that the abrupt change of the rotor resistance at $0.6 \mathrm{~s}$ causes great fluctuations and errors of estimation speed. After adding rotor resistance online estimation at $1.2 \mathrm{~s}$, the fluctuation of speed and errors are reduced to be equal to those before the rotor resistance change. Therefore, the effectiveness of estimation algorithm at low speed, medium speed, and high speed is verified.

\section{CONCLUSIONS}

This paper presents a method for speed and rotor resistance estimation of AC induction motor. EKF-based speed estimation method is sensitive to motor parameters, especially rotor resistance. Therefore, reactive powerbased MRAS is used to perform the rotor resistance online estimation. This paper gives the block diagram of speed and rotor resistance estimation for IFOC IM. A simulation model is constructed based on the block diagram. The simulation results verified the effectiveness of the proposed method at low, medium and high speeds, respectively.

\section{ACKNOWLEDGEMENTS}

The authors wish to acknowledge the financial support and motivation provided by the Primary Research and Development Plan of Shandong Province (2016ZDJS03A04), the Fundamental Research Funds for the Central Universities (HIT.NSRIF.201705) and the Natural Science Foundation of Shandong Province (ZR2017MEE011).

\section{REFERENCES}

1. Holtz, J., \& Quan, J. (2003). Drift- and parametercompensated flux estimator for persistent zerostator-frequency operation of sensorless-controlled induction motors. Industry Applications IEEE Transactions on, 39(4), 1052-1060.

2. Rowan, T. M., Kerkman, R. J., \& Leggate, D. (1991). A simple on-line adaption for indirect field orientation of an induction machine. IEEE Transactions on Industry Applications, 27(4), 720727.

3. De, S. R. L. A., Jacobina, C. B., Lima, A. M. N., \& Oliveira, A. C. (1997). Parameter sensitivity of mrac models employed in ifo-controlled ac motor drive.
IEEE Transactions on Industrial Electronics, 44(4), 536-545. 